\title{
UNA POLÉMICA SOBRE LA FOTOTERAPIA. LA APORTACIÓN DE UN OFTALMÓLOGO ESPAÑOL
}

\author{
Diego Peral \\ Doctor en Medicina - Profesor Asociado de Historia de la Medicina - Universidad de Extremadura \\ Jesús Fernández-Sabugal \\ Doctor en Medicina - Profesor Titular de Oftalmología - Universidad de Extremadura
}

\section{RESUMEN}

El Dr. Morón Salas ensayó experimentalmente la utilización de la luz como terapéutica para fotocoagulación del fondo de ojo.

Sus primeras experiencias comienzan en 1945, culminando con la defensa de su tesis doctoral. No publica sus resultados hasta 1950, tras haber leído una comunicación del Dr. Meyer-Schwickerath, que había conseguido éxito tratando desprendimientos de retina en humanos con luz solar.

Los autores estudian la posible prioridad del oftalmólogo español en el descubrimiento del método.

\section{SUMMARY}

The Dr. Moron Salas tested experimentally the utilization of the light as therapy for photocoagulation of the eye fund.

His first experiences begin in 1945, peaing with the defense of his doctoral thesis. It does not publish his results until 1950, after have read a communication of the Dr. Meyer-Schwickerath, that had obtained success trying retina detachings in human with sunlight.

We study the priority of spanish ophthalmologist over the discovery of this method

\section{INTRODUCCIÓN}

A mediados del siglo XIX, con el descubrimiento del oftalmoscopio, comienza la exploración del fondo de ojo. En 1854 von Graefe publica una descripción de las lesiones del fondo de ojo, en relación con el desprendimiento de retina. El tratamiento de esta patología ha sido eminentemente quirúrgico desde el siglo XIX hasta 


\section{DIEGO PERAL Y JESÚS FERNÁNDEZ-SABUGAL}

nuestros días. El planteamiento que realiza el Dr. Krewson en 1939 sobre la evolución del tratamiento de la separación de la retina es válido hoy:

\footnotetext{
“... have been conceived numerous and diverse treatments, all of which have passed through a long evolutionary process, beginning with frank empiricism and advancing to the present rational and scientific state."
}

El tratamiento etiológico comienza en el siglo XX con las afirmaciones de Go$\mathrm{nin}^{2}$, que considera al agujero retiniano como el factor clave en la aparición de este cuadro. En el comienzo existe diversidad de técnicas y variaciones sobre las mismas, que en el transcurrir de la investigación se van estandarizando hasta llegar hoy en día a la prevención del desprendimiento de retina mediante el cierre de las roturas retinianas con LASER antes de que se produzca el desprendimiento.

El tratamiento en las décadas de los años cuarenta y cincuenta sigue teniendo por objeto evacuar el líquido subretiniano y producir una corio-retinitis adhesiva en el lugar del desgarro retiniano utilizando diatermia ${ }^{3}$. En la primera década aparece una nueva idea de tratamiento del desprendimiento de retina, que consiste en cauterizar el desgarro de la retina utilizando la luz como medio terapéutico; el desarrollo de esta técnica culminará con la aparición y desarrollo de la tecnología del LASER.

En los años cuarenta dos oftalmólogos tendrán el pensamiento de utilizar la luz para cerrar o sellar el desgarro retiniano: el Dr. Meyer-Schwickerath y el Dr. Morón Salas. Poner de manifiesto las aportaciones a la fotocoagulación de este último, es el principal objeto de este trabajo.

\section{EL PERSONAJE}

En marzo de 1918 nace en Sevilla José Morón Salas. Su familia se traslada a Cuba cuando él tiene dos años y permanecen allí durante cinco años. El padre ejerció en la isla de oculista, inducido por su suegro que también era oftalmólogo y había residido durante algún tiempo en Santa Clara de Cuba. En 1925 Don José Morón Ruiz se instalará definitivamente en Sevilla, dejando su consulta de ultramar en manos de su primo José Ruiz Velasco, también oftalmólogo, que pasado el tiempo llegará a ser embajador en el Vaticano del Régimen de Fidel Castro.

1 KREWSON, W.E. (1939), «History of the surgical treatment of retinal separation» Archives of Ophthalmology. 22.: 292-312; p. 17.

2 KREWSON, W.E. (1939), p. 8.

3 CARRERAS Duran, B.; CARReras Matas, B. (1956). Introducción a la Oftalmología. Labor (ed.). Barcelona, p. 158. 
José Morón Salas comienza la carrera de medicina en Sevilla, pero ésta se ve interrumpida por la Guerra Civil Española. Sus destinos en la contienda estarán siempre relacionados con el mundo sanitario, siendo el último el de "Agregado a los Laboratorios de Fisiología de la Junta Técnica del Servicio de Defensa Química del Ejército del Sur"4. Concluidos sus estudios de medicina en 1941, su actividad profesional se centrará en dos campos: la asistencia a la Cátedra de Oftalmología de la Facultad de Medicina de Sevilla, donde será nombrado Ayudante de Clases Prácticas por el Profesor Díaz Domínguez, y la consulta junto con su padre. Debemos precisar que su apredizaje oftalmológico comenzó mucho antes de las fechas mencionadas, ya que acompañaba a su padre desde los doce años, observando y diagnosticando junto a él enfermedades oculares.

En 1946 defiende su tesis doctoral y se presenta posteriormente a la plaza de $\mathrm{Au}$ xiliar de Cátedra de Universidad, que no consigue.

En 1950 solicita una beca de un año de duración que oferta el Dr. Ramón Castroviejo, la cual obtiene, y se marcha a Nueva York, para ser su ayudante particular durante ese tiempo. El Dr. Castroviejo le propondrá que una vez finalizada la beca continue siendo su ayudante, para posteriormente ser su colaborador y socio. El Dr. Morón desestima su oferta de trabajo y decide volver a España para continuar su ejercicio profesional en la consulta con su padre, ante el requerimiento que éste le formula.

\section{SU HIPÓTESIS}

José Morón Salas, en 1945, durante la intervención de un desprendimiento de retina utilizando diatermia para cauterizar el desgarro a través de la esclera, que era el tratamiento habitual de la época, tuvo la idea de producir la quemadura que cerrara el desgarro retiniano utilizando la luz. A partir de aquí comienza a llevar a la práctica su hipótesis, que consiste en utilizar una fuente de luz que, a través de la pupila, llegue a la retina y produzca una quemadura similar a la de la diatermia. Esto se traducirá en la primera experiencia mundial conocida de fotocoagulación terapéutica en retina. Sus primeras experiencias son recogidas en la tesis doctoral que defiende en la Universidad de Madrid el 22 de Noviembre de $1946^{5}$, y publicadas en $1950^{6}$.

\footnotetext{
4 MORÓN SALAS, J. (1989). Autobiografia inconclusa. Inédita. Sevilla. p. 7.

5 MORÓN SALAS, J (1946). Sobre etiología y patogenia de los fototraumatismos retinianos. Tesis Doctoral Inédita. Universidad Complutense. Madrid.

6 MORÓN SALAS, J. (1950) «Obliteración de los desgarros retinianos por quemadura con luz». Arch. Soc. Oftalmol. Hispano-Americana. 10/6, 566-578.
} 


\section{DIEGO PERAL Y JESÚS FERNÁNDEZ-SABUGAL}

\section{LA POLÉMICA}

Se puede especular si sus estudios experimentales fueron anteriores a los llevados a cabo por el investigador alemán Meyer-Schwickerath, considerado mundialmente "padre" de la fotocoagulación, ya que fue el que desarrolló definitivamente la técnica, hasta conseguir resultados clínicamente útiles. En 1972 la prensa española se hacía eco de una polémica, que se podría seguir manteniendo en la actualidad, acerca de la primacía del español en el descubrimiento de la utilización terapéutica del efecto fotocoagulante de la luz en la retina, publicada en su Tesis Doctoral en 19467.

También el Dr. Meyer-Schwickerath refiere en sus trabajos que la idea de la fotocoagulación surgió en él en $1946^{8 \cdot 9}$, aunque no publicó hasta $1949^{10}$.

Castroviejo, por su parte, en carta personal dirigida a Morón sobre el escrito que publica Meyer-Schwickerath en 1967 sostiene la primacía de aquél sobre el germano, considerando que se inspiró en los trabajos del médico sevillano ${ }^{11}$.

Olivella Casals, contacta con Meyer-Schwickerath y viaja a Bonn en diciembre de 1955 para conocer directamente sus experiencias ${ }^{12}$. Basándose en los datos que le proporciona, y con algunas modificaciones añadidas por el ingeniero óptico $\mathrm{C}$. Garrigosa, construye el segundo fotocoagulador clínicamente útil que existía en aquel momento en el mundo ${ }^{13}$.

La fotocoagulación alcanzará su máximo con el desarrollo en 1960 por Maiman ${ }^{14}$ de la luz LASER, que es monocromática, altamente direccional y de gran luminosidad, y su aplicación a la oftalmología se realiza en 1962.

7 Diario Hierro. Bilbao. 18 de enero de 1972. N $^{\circ} 12680$. p. 8.

8 MEYER-SCHWICKERATH, G. (1967)«History and development of photocoagulation», Am J Ophthamol, 63 (6), 1812-1814.

9 MEYER-SCHWICKERATH, G. (1989) «The history of photocoagulation.» Aust N Z J Ophthalmol. $17(4), 427-434$.

10 MeYer-SChwiCKerath, G. (1949) «Koagulation der Netzhaut mit Sonnenlicht.» Ber. Dtsch. Ophthal. Ges. 55: 256-259.

11 CASTROVIEJO, R. (3-7-1967) New York. (Carta personal).

12 Olivella CASAlS, A. (1959) La fotocauterización como tratamiento de algunas afecciones del fondo ocular y del iris. Barcelona, p. 4.

13 Olivella CaSAlS, A. (1968). La Fotocoagulación como tratamiento en Oftalmología. Barcelona. p. 30 .

14 ZARAgOZA, J.R. (1992), Física e instrumentación médicas. Ed. Masson. Barcelona. p. 571. 


\section{EL MÉTODO MORÓN}

En su primera experiencia, nuestro médico dilató la pupila del ojo de un conejo, y trató de producir una quemadura en la retina-coroides, observando el fondo de ojo con luz solar y a imagen inversa ${ }^{15}$. Este primer y desordenado ensayo, extremadamente cáustico al prolongarse "unos minutos" 16 , transformó la mitad del fondo de ojo en una masa blanca y prominente que al cabo de unos días dejó una cicatriz extensa y similar a la que aparecía cuando se realizaba coagulación diatérmica.

A continuación Morón prosigue sus experiencias prescindiendo del oftalmoscopio indirecto y la lupa, dejando que la luz solar actuase directamente sobre la retina (en un ojo emétrope los rayos que llegan paralelos focalizan en la retina) (Figura 1), con el objeto de evitar la perdida de energía que se produce al atravesar estos medios ópticos. Esta experiencia conlleva la dificultad de mantener al animal inmóvil para que la luz focalice en el mismo punto siempre.

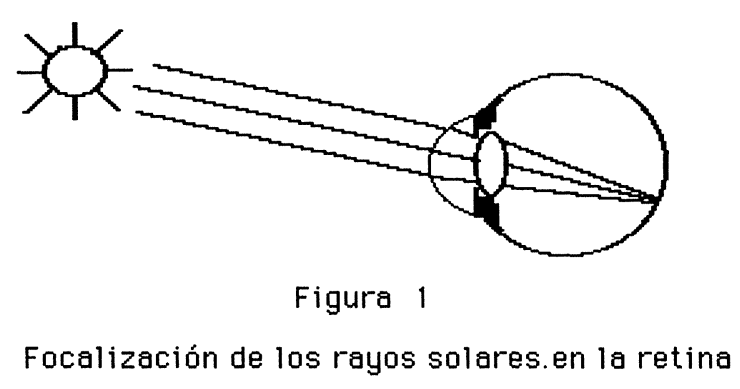

EI siguiente paso fue tratar de sustituir la fuente de luz natural por una fuente artificial con la intensidad suficiente para conseguir los efectos deseados en el fondo de ojo. Tras varios e ineficaces intentos, se hizo construir "un arco voltaico de 15 amperes" 17 con el cual llevó a cabo desde entonces todas sus experiencias.

Los resultados en conejos fueron exitosos, consiguiendo fotocoagulaciones eficaces y controladas en el fondo de ojo. Esto le llevó a ensayar en cuatro ojos humanos con desprendimiento de retina (el fin último de la técnica era "cauterizar" los desgarros retinianos). La técnica empleada en humanos consistió en la dilatación, lo más

15 Se realiza utilizando un oftalmoscopio y una lupa.

16 MORÓN SALAS, J. (1950) «Obliteración de los desgarros retinianos por quemadura con luz». Arch. Soc. Oftalmol. Hispano-Americana. 10/6, 566-578.

17 MORÓN SAlaS, J. (1964) «Pasado, presente y futuro de la fotocoagulación». Academia Médico Quirúrgica Provincial. Vigo. Tomo VI, N"2 (Sesión del 15 de mayo de 1964). 
posible, de la pupila, localización del desgarro retiniano oftalmoscópicamente y colocaciớn del arco voltaico en el lugar del observador (con lo cual la luz iría en la dirección del desgarro retiniano) para comenzar la irradiación de la zona afecta, utilizando tiempos de exposición 60 veces superiores a los que fueron eficaces en los conejos (la intensidad es constante, pues es la que proporciona el arco voltaico de que dispone).

Esta experiencia fue un completo fracaso, pero sin desanimarse probó aún su aparato en dos retinas humanas sin desprendimento. Uno de los ojos era ciego por una atrofia del nervio óptico y el otro caso era un ojo de un voluntario sano. En ninguno de estos dos casos consiguió producir quemadura en el fondo de ojo, ensayando entonces una fuente luminosa más concentrada.

Los resultados obtenidos se exponen en las tablas I, II y III.

Tabla I

Experiencias de coagulación de fondo con luz solar

\begin{tabular}{|c|c|c|c|c|c|}
\hline Núm & Fecha & Hora & Duración & Filtro & Resultado \\
\hline $\begin{array}{l}1 \\
2 \\
3 \\
4 \\
5 \\
6 \\
7 \\
8 \\
9\end{array}$ & $\begin{array}{l}24 / 2 / 46 \\
25 / 2 / 46 \\
2 / 3 / 46 \\
6 / 3 / 46 \\
2 / 3 / 46 \\
24 / 2 / 46 \\
7 / 3 / 46 \\
20 / 3 / 46 \\
20 / 3 / 46\end{array}$ & $\begin{array}{l}13 \\
14,30 \\
14,30 \\
14 \\
14,30 \\
16,30 \\
15 \\
14,30 \\
14,30\end{array}$ & $\begin{array}{l}5 \text { minutos } \\
1 \text { minuto } \\
5 \text { minutos } \\
2 \text { minutos } \\
5 \text { minutos } \\
10 \text { minutos } \\
5 \text { minutos } \\
5 \text { minutos } \\
3 \text { minutos }\end{array}$ & $\begin{array}{l}\text { Ninguno } \\
\text { Ninguno } \\
\text { Vidrio } \\
\text { Agua y Vidrio } \\
\text { Solución de yodo y mica } \\
\text { Solución de yodo y mica } \\
\text { Mica } \\
\text { Violeta de genciana y vidrio } \\
\text { Sulfato de cobre y vidrio }\end{array}$ & $\begin{array}{l}\text { Positivo } \\
\text { Positivo } \\
\text { Positivo } \\
\text { Positivo } \\
\text { Negativo } \\
\text { Negativo } \\
\text { Positivo } \\
\text { Negativo } \\
\text { Negativo }\end{array}$ \\
\hline
\end{tabular}

Tabla II

Experiencias de coagulación de fondo con luz de arco

\begin{tabular}{|l|c|l|l|l|}
\hline Núm. & Amperes & Duración & \multicolumn{1}{|c|}{ Filtro } & Resultado \\
\hline 10 & 4 & $18 \mathrm{~min}$. & Vidrio & Negativo \\
11 & 4 & $15 \mathrm{~min}$. & Vidrio & Negativo \\
12 & 8 & $1 \mathrm{~min}$. & Vidrio y agua. Rayos convergentes & Negativo \\
13 & 8 & $1 \mathrm{~min}$. & Vidrio y agua. Rayos convergentes & Negativo \\
14 & 8 & $3 \mathrm{~min}$ & Vidrio y agua & Positivo \\
15 & 8 & $1,5 \mathrm{~min}$ & Vidrio y agua & Positivo \\
16 & 8 & $1 \mathrm{~min}$. & Vidrio y agua & Positivo \\
17 & 8 & $1 \mathrm{~min}$. & Vidrio y agua & Positivo \\
\hline
\end{tabular}


UNA POLÉMICA SOBRE LA FOTOTERAPIA. LA APORTACIÓN DE UN OFTALMÓLOGO ESPAÑOL

Tabla III

Experiencias de coagulación de fondo con luz de arco concentrada

\begin{tabular}{|l|c|c|c|c|}
\hline Núm. & Amperes & Duración & Filtro & Resultado \\
\hline 18 & 10 & $1 \mathrm{~min}$. & Agua y vidrio & Positivo \\
19 & 10 & $30 \mathrm{seg}$. & Agua y vidrio & Positivo \\
20 & 11 & $30 \mathrm{seg}$. & Agua y vidrio & Positivo \\
21 & 11 & $15 \mathrm{seg}$. & Agua y vidrio & Positivo \\
22 & 10 & $15 \mathrm{seg}$. & Agua y vidrio & Positivo \\
23 & 11 & $5 \mathrm{seg}$. & Agua y vidrio & Positivo \\
24 & 10 & $2 \mathrm{seg}$. & Agua y vidrio & Negativo \\
25 & 10 & $1 \mathrm{~min} . \mathrm{y} 30 \mathrm{seg}$. & Vidrio rojo y agua & Negativo \\
26 & 10 & $2 \mathrm{~min} . \mathrm{y} 30 \mathrm{seg}$. & Vidrio rojoy agua & Negativo \\
\hline
\end{tabular}

Entre las tablas II y III la diferencia entre los ensayos estriba en que en la segunda se concentra la luz para no desperdiciar energía. Esto se consigue modificando el radio de curvatura del espejo hasta un punto tal que todos los rayos formen un foco antes de llegar al ojo. Por delante del foco (donde los rayos comienzan a diverger) se coloca una lente convergente que transformará los haces de luz en paralelos que es la forma en que deben de llegar al ojo para focalizar en la retina, en un ojo emétrope.(Figuras 2 y 3 ).

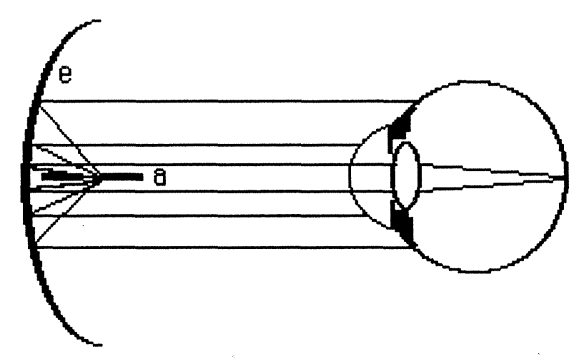

Figura 2

La luz procedente del cráter del arco a, es reflejada en haz de rayos paralelos por el espejo e, para llegar al ojo. 
DIEGO PERAL Y JESÚS FERNÁNDEZ-SABUGAL

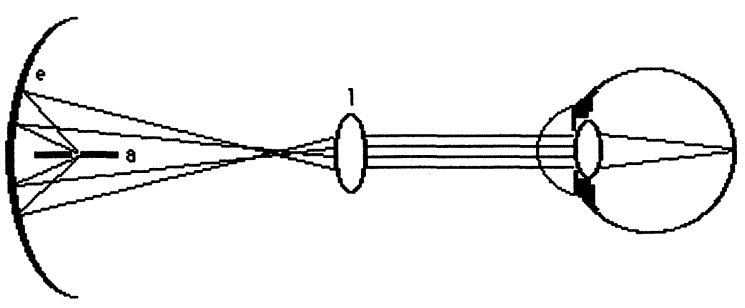

Figura 3

La luz del arco a es reflejada por el espejo e en un haz convergente. La lente 1 envía la luz hacia el ojo en haz de rayos paralelos. Este haz viene constituido por toda la luz que se reflejó en el espejo.

No observa alteraciones en el cristalino en ninguno de los ojos fotocoagulados en los conejos, ni en las experiencias realizadas en humanos, lo que muestra que la técnica no es yatrogénica para las estructuras transparentes del ojo.

\section{CONCLUSIÓN}

El Dr. Meyer-Schwickerath en sus primeras publicaciones cita al Dr. Morón Salas como otro precursor de la idea de la fotocoagulación ${ }^{18}{ }^{19}$. Posteriormente desaparece este nombre en sus trabajos, hecho que llama la atención, incluso también para el Dr. Castroviejo en ese mismo momento.

$\mathrm{Al}$ realizar una revisión sobre la historia de la fotocoagulación, el Dr. Morón no aparece citado por ningún autor extrajero, habiendo quedado en el olvido, debido a que todos se basan en los trabajos de Meyer-Schwickerath. Sirva como ejemplo el artículo de Lawrence A. Raymond ${ }^{20}$.

Pero cabe preguntarse: ¿de quién fue la prioridad?. Lo único claro es que estos dos investigadores tuvieron la misma idea, de forma más o menos simultánea en el tiempo, y que es el oftalmólogo alemán el que la logra culminar, quedando las aportaciones del Dr. Morón a los comienzos de la fotocoagulación en el olvido, excepto

18 MEYER-SCHWICKERATH, G. (1954) «Lichtkoagulation. Eine Methode zur Behandlung und Wertung der Netzhautablösung» Graefes Archiv für Ophthalmologie, Bd. 156, S.2-34.

19 MEYER-SCHWICKERATH, G. (1959) Lichtkoagulation. Stuttgart. p. 2.

20 RAYMOND, L.A. (1967) «Historical perspectives on photocoagulation». Surv. Ophthalmol. 21 (6), 501-505. 
UNA POLÉMICA SOBRE LA FOTOTERAPIA. LA APORTACIÓN DE UN OFTALMÓLOGO ESPAÑOL

para autores españoles como Casanovas (1975) ${ }^{21}$ y Corcóstegui (1988) 22 que datan las experiencias de Morón Salas en 1949 y 1950 respectivamente, aunque ya señalamos que él desarrolló su fase experimental entre 1945 y 1946.

A nuestro entender los trabajos del Dr. Morón, aunque sus estudios estén todos inconclusos, marcan el comienzo de una nueva etapa en la oftalmología: es el primer investigador que utiliza la luz para producir quemaduras terapéuticas en el fondo de ojo.

Es un investigador solitario y esto conlleva una falta de medios económicos, infraestructura y soporte institucional. Estos factores le condicionan de una forma tal que no logra culminar el trabajo empezado.

Sirva este trabajo para rescatar del olvido su figura y mostrar su aportación a un capítulo importante de la oftalmología.

21 CASANOVAS, J. (1975). Oftalmología: patología y clínica quirúrgica, (Cood.) Laín, P: Historia Universal de la Medicina. Tomo VII. Barcelona. p. 362.

22 GómeZ-Ulla, F; CORCóstegui, B (1988). Angiografia fluoresceínica y Láser. Santiago de Compostela, p. 52. 